\title{
Molecular characterization of alterations in the intestinal microbiota of patients with grade 3 hypertension
}

\author{
NOSHEEN MUSHTAQ ${ }^{1}$, SAFDAR HUSSAIN ${ }^{2,3}$, SIRUO ZHANG $^{1}$, LU YUAN $^{1}$, \\ HUAN LI ${ }^{1}$, SHAKIR ULLAH $^{1}$, YAN WANG $^{4}$ and JIRU XU ${ }^{1}$ \\ Departments of ${ }^{1}$ Pathogenic Microbiology and Immunology, and ${ }^{2}$ Molecular Biology and Biochemistry, \\ School of Basic Medical Sciences, Xi'an Jiaotong University, Xi'an, Shaanxi 710061, P.R. China; \\ ${ }^{3}$ Centre for Applied Molecular Biology (CAMB), University of The Punjab, Lahore, Punjab 53700, Pakistan; \\ ${ }^{4}$ Clinical Laboratory, Xi'an No. 5 Hospital, Xi'an, Shaanxi 710061, P.R. China
}

Received October 30, 2018; Accepted May 30, 2019

DOI: $10.3892 / \mathrm{ijmm} .2019 .4235$

\begin{abstract}
Hypertension has become a major risk factor for many diseases, including cardiovascular, cerebrovascular and kidney disorders. It has been reported that the composition of human gut microbiota is changed during the progression of cardiovascular and kidney diseases. The current study aimed to qualitatively and quantitatively compare the composition of gut microbiota between patients with hypertension and healthy controls. Fecal samples were collected from 50 patients diagnosed with grade 3 hypertension and 30 healthy controls. Touchdown PCR-denaturing gradient gel electrophoresis with primers specifically targeting the $\mathrm{V} 3$ region of $16 \mathrm{~S}$ ribosomal RNA, and quantitative PCR, were performed to characterize all the samples. High-throughput sequencing of the V3-V4 regions was performed on 30 randomly selected samples. By comparing diversity and richness indices, the gut microbiome of the hypertensive individuals was found to be more diverse than that of the healthy controls. Among the main bacterial phlya that reside in the gut, Bacteroidetes, Firmicutes and Proteobacteria were dominant in all the samples; however the Firmicutes to Bacteroidetes ratio was variable, with a significant increase in the patients with hypertension compared with the healthy control group. In addition, at the genus level, there was an increased abundance of Prevotella_9, Megasphaera, Parasutterella and Escherichia-Shigella in patients with hypertension, while Bacteroides and Faecalibacterium were decreased. These results suggested that the human gut microbiota is altered in hypertension, and understanding the mechanism of these changes in microbial composition may
\end{abstract}

Correspondence to: Professor Jiru Xu, Department of Pathogenic Microbiology and Immunology, School of Basic Medical Sciences, Xi'an Jiaotong University, 76 Yanta West Road, Xi'an, Shaanxi 710061, P.R. China

E-mail: xujiru@mail.xjtu.edu.cn

Key words: hypertension, gut microbiota, denaturing gradient gel electrophoresis, quantitative PCR, high-throughput sequencing open up new insights, and help to treat hypertension and other related diseases.

\section{Introduction}

Hypertension has become a major factor in the global burden of disease and mortality, contributing to millions of mortalities every year caused by coronary heart disease and stroke (1), peripheral vascular disease, heart failure, chronic kidney disease, cognitive dysfunction and dementia (2). Hypertension remains a critical public health problem despite many developments in the field, and advancements in the diagnosis, management and control of the disease. Despite changes in lifestyle and advancements in pharmacotherapy, the prevalence of hypertension remains high. A survey in 2010 revealed that $\sim 31 \%$ of the world's population have hypertension, with more than one billion of these from developing countries (3). Maintaining homeostatic blood pressure (BP) is a complex process regulated by multiple factors, including genetic factors, environmental factors and endocrine regulation in the kidneys. Studies have suggested that human gut microbiota may have a role in the regulation of BP and the pathogenesis of hypertension (4-6) by secreting a variety of microbe-derived bioactive metabolites, such as short-chain fatty acids $(7,8)$.

Metabolomics has identified new pathogenic pathways involved in BP regulation $(9,10)$. However, identifying the causes of hypertension remains challenging due to the heterogenic and complex nature of the disease. The adult human gut microbiome is diverse, being made up of trillions of microorganisms. Generally it is dominated by four phyla: Firmicutes, Bacteroidetes, Actinobacteria and Proteobacteria (11). An imbalance in the composition of normal gut microbiota is commonly known as dysbiosis (12). Although all healthy gut microbiota have yet not been fully characterized, increasing evidence from various reports suggests that changes in the ratio of microbial communities, such as Firmicutes and Bacteroidetes (the $\mathrm{F} / \mathrm{B}$ ratio), can potentially be used as biomarkers identify pathological conditions $(13,14)$. A study using animals and human patients showed that decreased microbial richness, evenness and diversity, and an increased 
$\mathrm{F} / \mathrm{B}$ ratio, resulting in gut microbiota dysbiosis, were associated with hypertension (1).

Several studies have reported the effects of probiotics on BP regulation (15-17). A meta-analysis of nine randomized trials showed that a daily dose of $\geq 109$ colony-forming units probiotics significantly decreased both systolic BP (SBP) and diastolic BP (DBP) in human patients with hypertension (15). These data indirectly suggest that gut microbiota may have an important role in maintaining BP homeostasis, and that an imbalance or any change in microbiota composition may potentially be involved in the development of hypertension. Many other studies have suggested that an imbalance in the gut microbiota (or dysbiosis) can result in chronic inflammatory diseases, such as inflammatory bowel disease (encompassing ulcerative colitis and Crohn's disease), asthma, allergies and infectious diseases $(18,19)$, systemic diseases such as diabetes (20), and other pathological conditions, including obesity (21) and malnutrition (22). High-throughput sequencing technologies and other parallel developments in nongenomic techniques have made it possible to elucidate the notable role of gut microbiota in human health and disease.

In the current study, standard metagenomic techniques, such as high-throughput sequencing along with touchdown PCR-denaturing gradient gel electrophoresis (DGGE) and quantitative PCR (qPCR), were used to characterize gut microbial diversity in patients diagnosed with grade 3 hypertension, with healthy volunteers as controls.

\section{Materials and methods}

Study participants and design. The present study included 50 hypertensive patients ( 28 males and 22 females). All of the patients were between 40 and 75 years of age and had grade 3 hypertension [according to the World Health Organization BP classification (Table SI)]. Patients were examined and excluded if they had any other acute or chronic inflammatory or infectious disease. The control group included 30 healthy volunteers (16 males and 14 females, aged 40-70 years). All of the healthy volunteers were in good health with no history of hypertension, or any other cardiovascular or chronic metabolic disease. None of the patients or healthy individuals had a history of taking any antibiotics, probiotics or prebiotics within the preceding 30 days at the time of sampling. Informed written consent was obtained from all participants in the study. The present study was performed with the approval of the Ethical Committee of Xi'an Jiaotong University School of Medical Sciences, under the guidelines of the World Medical Association and the Declaration of Helsinki. Fecal samples were collected in sterile cups and were stored immediately at $-80^{\circ} \mathrm{C}$ until the DNA extraction was carried out.

Biochemical measurements (Data S1). Blood pressure and lipid profile analysis data for each patient were collected from the Cardiology Department of the 1st Affiliated Hospital of Xi'an Jiaotong University (Table SII). The mean values of each analysis are summarized in Table I.

DNA extraction. Bacterial DNA was extracted using the Qiagen QIAamp MiniStool kit (Qiagen $\mathrm{GmbH}$ ), according to the manufacturer's instructions. The concentration of extracted DNA was estimated via the absorbance at $260 \mathrm{~nm}$ (A260), and the purity was determined by calculating the A260/A280 ratio with a NanoDrop spectrophotometer (Thermo Fisher Scientific, Inc.).

PCR-DGGE analysis. Universal primers for the V3 region of the 16S ribosomal (r)RNA gene were used to amplify the bacterial DNA of the study samples, using the fecal DNA as a template for PCR-DGGE analysis. Each $50 \mu \mathrm{l}$ PCR reaction mixture contained $1 \mu \mathrm{l}$ of each primer, $1 \mu \mathrm{l}$ dNTPs $(10 \mathrm{mM})$, $5 \mu \mathrm{l} \mathrm{MgCl}_{2}(25 \mathrm{mM}), 5 \mu \mathrm{l} 1 \mathrm{X}$ buffer, $0.4 \mu \mathrm{l} \mathrm{Taq}$ DNA polymerase (Takara Bio, Inc.) and $2 \mu \mathrm{l}$ fecal DNA. PCR amplification was performed in an automated thermocycler (ABI2720; Applied Biosystems; Thermo Fisher Scientific, Inc.) using a touchdown PCR program in order to increase the PCR reaction specificity. The PCR thermal profile conditions were as follows: Initial denaturation at $95^{\circ} \mathrm{C}$ for $5 \mathrm{~min}$, followed by 35 cycles of $1 \mathrm{~min}$ denaturation at $95^{\circ} \mathrm{C}$, annealing at $65^{\circ} \mathrm{C}$ for $1 \mathrm{~min}$ and extension at $72^{\circ} \mathrm{C}$ for $1 \mathrm{~min}$ (23). The PCR products were electrophoresed in $1.5 \%$ agarose gel stained with ethidium bromide in 1X Tris-acetate EDTA (TAE) buffer for visualization under UV illumination. The primer sequences used for PCR amplification are presented in Table II.

DGGE was executed using the DCode ${ }^{\mathrm{TM}}$ Universal Mutation Detection System (Bio-Rad Laboratories, Inc.). Sequence-specific separation of the amplicons was performed using 10\% (w/v) polyacrylamide (acrylamide-bis, 37.5:1) gels in 1X TAE buffer, containing 30-65\% linear denaturing gradient. Electrophoresis was performed at a constant voltage of $90 \mathrm{~V}$ at $60^{\circ} \mathrm{C}$ for $13 \mathrm{~h}$. The gels were stained with $5 \mu \mathrm{g} / \mathrm{ml}$ ethidium bromide solution for $30 \mathrm{~min}$, washed with deionized water and then viewed under a Bio-Rad Gel Doc 2000 (Bio-Rad Laboratories, Inc.). Comparison of DGGE profiles in the different gels was performed using a standard reference DNA Marker (DL2000; Takara Bio, Inc.) The distinct, prominent and frequent bands were cut from the gels, washed with RNAse-free water, resuspended in $20 \mu \mathrm{l}$ RNAse-free water and stored at $4^{\circ} \mathrm{C}$ overnight. PCR was performed again under the aforementioned conditions using $\mathrm{V} 3$ region primers 341F/534R, this time without a GC clamp. Re-amplified PCR products were sequenced using the ABI 3500xL system (Applied Biosystems; Thermo Fisher Scientific, Inc.). Sequences were scanned using the Basic Local Alignment Search Tool (https://blast.ncbi.nlm.nih.gov/Blast.cgi) and Seqmatch (http://rdp.cme.msu.edu/seqmatch/seqmatch_intro. jsp) software to categorize the species or genera.

Statistical analysis of DGGE. Bacterial diversity was evaluated by band number and band intensities in the DGGE profiles using Quantity One software (version 4.6.2; Bio-Rad Laboratories, Inc.). The diversity of taxa in the fecal microbiota was determined by Shannon-Weaver index $\left(\mathrm{H}^{\prime}\right)$. Similarity scoring and cluster analysis of DGGE profiles was performed using the UPGMA method based on the Dice similarity coefficient (24). SPSS software (version 20; SPSS, Inc.) was used for the nonparametric statistical analysis. Results are expressed as the mean \pm standard deviation.

qPCR analysis. To calculate the absolute copy number of Bacteroides spp., Prevotella spp., Clostridium spp. and Escherichia coli within the test samples, qPCR was 
Table I. Characteristics of study participants.

\begin{tabular}{lccc}
\hline Characteristic & HTN $(\mathrm{n}=50)$ & $\mathrm{CG}(\mathrm{n}=30)$ & P-value \\
\hline Sex (male/female) & $28 / 22$ & $16 / 14$ & - \\
Age, years & $62.5 \pm 10.4$ & $60.5 \pm 11$ & 0.47 \\
Weight, $\mathrm{kg}$ & $68.4 \pm 8.01$ & $65.3 \pm 5.9$ & 0.07 \\
$\mathrm{SBP}, \mathrm{mmHg}$ & $180.34 \pm 15.44$ & $122.83 \pm 7.6$ & $<0.0001$ \\
DBP, $\mathrm{mmHg}$ & $106.88 \pm 10.1$ & $79.63 \pm 6.8$ & $<0.0001$ \\
FBG, $\mathrm{mmol} / \mathrm{l}$ & $5.05 \pm 0.87$ & $4.22 \pm 0.64$ & $<0.0001$ \\
TC, $\mathrm{mmol} / \mathrm{l}$ & $4.07 \pm 0.82$ & $4.34 \pm 0.90$ & 0.17 \\
TG, $\mathrm{mmol} / \mathrm{l}$ & $1.48 \pm 0.55$ & $1.10 \pm 0.30$ & $<0.005$ \\
HDL, $\mathrm{mmol} / \mathrm{l}$ & $1.04 \pm 0.24$ & $1.10 \pm 0.21$ & 0.21 \\
LDL, $\mathrm{mmol} / \mathrm{l}$ & $2.26 \pm 0.63$ & $1.87 \pm 0.53$ & $<0.05$ \\
\hline
\end{tabular}

Data for age, weight, SBP, DBP, FGB, TG, TC, HDL and LDL are presented as the mean \pm SD. P-values for age, weight, SBP, DBP, FGB, HDL, LDL, TG and TC were calculated using Student's t-test. SBP, systolic blood pressure; DBP, diastolic blood pressure; FBG, fasting blood glucose; HDL, high density lipoprotein; LDL, low density lipoprotein; TG, total triglyceride; TC, total cholesterol.

performed with a QuantiFast SYBR-Green PCR kit (Qiagen $\mathrm{GmbH}$ ) using the StepOne v2.3 real-time PCR detection system (Applied Biosystems; Thermo Fisher Scientific, Inc.). The reaction mixture $(20 \mu \mathrm{l})$ was composed of $10 \mu \mathrm{l}$ 2X SYBR-Green PCR mix (Takara Bio, Inc.), $0.8 \mu \mathrm{l}$ of each specific primer (Table II), $2 \mu 1$ sample DNA and $6.4 \mu 1$ sterile deionized water. The total copy number within each sample was calculated by comparing with a standard curve prepared from serially diluted plasmid DNA (of known concentration) ranging from $10^{2}$ to $10^{8}$ copies/g of feces. The qPCR data were analyzed using the absolute quantification method (25). For each bacterial group, a partial 16S rRNA gene sequence was amplified with PCR primers (Table II), and was subsequently cloned into the pMD18-T vector using the $\mathrm{pMD}^{\mathrm{TM}} 18-\mathrm{T}$ Vector Cloning kit (Takara Biotechnology Co., Ltd.), as described previously (26). Negative controls, containing all the elements of the reaction mixture except the template DNA, were run along with each reaction. The resultant bacterial populations were expressed as $\log 10$ bacterial replica counts in $1 \mathrm{~g}$ of the fecal mass. Triplicate samples were used in each experiment and the mean values were obtained. Student's t-test was used to calculate the significance $(\mathrm{P}<0.05)$ between the two groups. Results are expressed as the mean \pm standard deviation.

High-throughput sequencing. A total of 30 fecal samples were randomly selected for high-throughput sequencing and meta-analysis (20 samples from the hypertensive group and 10 from the control group). The V3-V4 region of the 16S rRNA gene was amplified using specific primers: 515-F (5'-GTGCCA GCMGCCGCGGTAA-3'), 806-R (5'-GGACTACHVGGG TWTCTAAT-3'), to create the amplicon libraries (27). The libraries were sequenced on an Illumina HiSeq 2500 platform according to the manufacturer's instructions. The raw data obtained were screened and assembled using the QIIME (28) and FLASH (29) software packages. The bacterial sequencing data were grouped into operational taxonomic units (OTUs) at a 97\% similarity level against the Greengenes database (30) using UCLUST (31) version 1.2.22q. All of the reads that failed to match the reference sequences were excluded; this is referred to as a 'closed reference' approach to clustering. A representative sequence for each OTU was screened for further annotation. For each representative sequence, the Greengenes database was used based on the RDP classifier (version 2.2) algorithm (32). The $\alpha$-diversity analysis, including Shannon and Simpson diversity index, Chaol algorithm, alternating conditional expectations (ACE) algorithm and Good's coverage, was performed with QIIME (version 1.7.0) and displayed using R software (version 2.15.3) (33). Student's $\mathrm{t}$-test was used to calculate the significant differences in $\alpha$-diversity between the two groups. $\mathrm{P}<0.05$ was considered to indicate a statistically significant difference. Linear discriminant analysis along with effect size measurements was used to examine the differentially significant taxa at each level. The relative abundances of phyla, families, genera and species in each sample were calculated and compared between the two groups. All statistical analyses were carried out using SPSS software (version 20).

\section{Results}

Biochemical measurements. The biochemical variables analyzed are shown in Table I. Briefly, the lipid profile data were different between the groups (hypertensive patients vs. healthy controls). Triglycerides, fasting blood glucose and low-density lipoprotein levels were significantly higher in the patients with hypertension compared with healthy controls $(\mathrm{P}<0.05)$. There was no significant difference in total cholesterol and high-density lipoprotein between the two groups. However, the SBP and DBP were markedly higher in the patients with hypertension.

DGGE profile analysis of the gut microbial communities. The results from DGGE analysis are shown in Fig. 1A. The DGGE profile band number and the Shannon diversity index $\left(\mathrm{H}^{\prime}\right)$ analysis, used to estimate the gut microbial diversity within the two groups, are presented in Table III. The similarity levels of DGGE profiles, measured by the Dice similarity coefficient and unweighted pair group method with arithmetic mean dendrogram, are shown in Fig. 1B. As shown in Table III, the intragroup similarity of the hypertensive group was significantly higher than the healthy control group $(\mathrm{P}<0.05)$. Comparing all the samples, the intergroup similarity index was lower than the intragroup similarity index. These results demonstrated that the gut microbiota of the hypertension group were different from the healthy control group. Dominant bands from different positions within the DGGE profiles were further analyzed by sequencing. Bacteroidetes and Firmicutes appeared to be the dominant bacterial phyla within both study groups (Table SIII).

Species-specific qPCR analysis. qPCR analysis was performed to quantify the changes in Bacteroides spp., Prevotella spp., Clostridium spp. and Escherichia coli in fecal samples from the two groups. There was a significant increase in Clostridium spp. and Prevotella spp. in the hypertension group compared with the healthy controls $(\mathrm{P}<0.05$; Table IV). E. coli was also increased in the hypertension group, but was not significantly 
Table II. PCR primers used in the study.

\begin{tabular}{|c|c|c|c|c|c|c|}
\hline $\begin{array}{l}\text { Author, } \\
\text { year }\end{array}$ & $\begin{array}{l}\text { Analysis } \\
\text { type }\end{array}$ & Target gene & & Primer sequence $\left(5^{\prime}-3^{\prime}\right)$ & $\begin{array}{l}\text { Product } \\
\text { size, bp }\end{array}$ & (Refs.) \\
\hline Muyzer et al, 1993 & PCR-DGGE & $\begin{array}{l}\text { Bacterial 16s rRNA } \\
\text { gene V3 region }\end{array}$ & $\begin{array}{l}341 \mathrm{~F}^{\mathrm{a}} \\
534 \mathrm{R}\end{array}$ & $\begin{array}{l}\text { CCTACGGGAGGCAGCAG } \\
\text { ATTACCGCGGCTGCTGG }\end{array}$ & 193 & (23) \\
\hline Huijsdens et al, 2002 & qPCR & Bacteroides & $\begin{array}{l}\text { Bac F } \\
\text { Bac R }\end{array}$ & $\begin{array}{l}\text { AAGGGAGCGTAGATGGATGTTTA } \\
\text { CGAGCCTCAATGTCAGTTGC }\end{array}$ & 193 & (68) \\
\hline Matsuki et al, 2002 & & Prevotella & $\begin{array}{l}\text { Pre F } \\
\text { Pre R }\end{array}$ & $\begin{array}{l}\text { ACAGTAAACGATGGATGCC } \\
\text { GGTCGGGTTGCAGACC }\end{array}$ & 513 & (69) \\
\hline Bartosch et al, 2004 & & Clostridium & $\begin{array}{l}\text { Clos F } \\
\text { Clos R }\end{array}$ & $\begin{array}{l}\text { CGGTACCTGACTAAGAAGC } \\
\text { AGTTTGATTCTTGCGAACG }\end{array}$ & 429 & (70) \\
\hline Bartosch et al, 2004 & & Escherichia coli & $\begin{array}{l}\text { E. coli } \mathrm{F} \\
\text { E. coli } \mathrm{R}\end{array}$ & $\begin{array}{l}\text { CATTGACGTTACCGCAGAAGAAGC } \\
\text { CTCTACGAGACTCAAGCTTGC }\end{array}$ & 190 & $(70)$ \\
\hline
\end{tabular}

${ }^{\text {aA }}$ 5' GC-clamp (CGCCCGCCGCGCGCGGCGGGCGGGGCGGGGGCACGGGGGG) was added for DGGE analysis. F, forward; R, reverse; DGGE, denaturing gradient gel electrophoresis; qPCR, quantitative PCR.
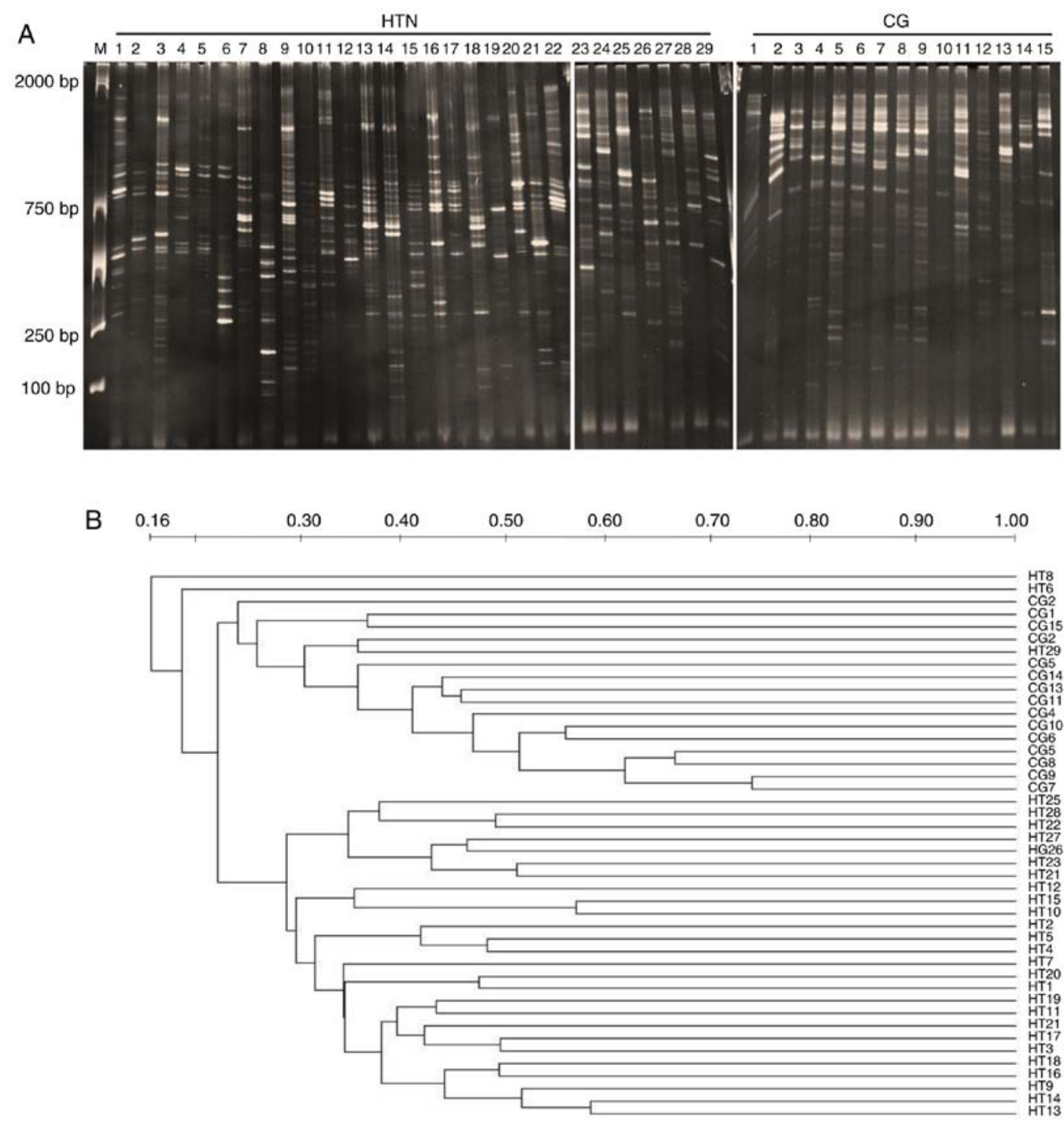

Figure 1. DGGE analysis of the study groups. (A) DGGE profiles of the two groups obtained from PCR-DGGE analysis using the universal primer for the V3 region of 16S ribosomal RNA. Lane 'M' represents the reference marker DL2000. (B) Clustering of DGGE profiles obtained with universal primer (V3) of the HTN group and CG using the Dice coefficient and the unweighted pair group method with the arithmetic mean. DGGE, denaturing gradient gel electrophoresis; HTN, hypertension; CG, control group. 
Table III. Microbiota diversity and similarity of the study groups.

\begin{tabular}{|c|c|c|c|}
\hline \multirow[b]{2}{*}{ Analysis } & \multicolumn{2}{|c|}{ Group } & \multirow[b]{2}{*}{ P-value } \\
\hline & HTN (n=29) & $\mathrm{CG}(\mathrm{n}=15)$ & \\
\hline \multicolumn{4}{|l|}{ Microbiota diversity } \\
\hline DGGE bands ${ }^{\mathrm{a}}($ mean $\pm \mathrm{SD})$ & $21.8 \pm 5.85$ & $16.3 \pm 4.31$ & 0.22 \\
\hline Shannon index ${ }^{b}\left(\right.$ mean $\left.H^{\prime} / H^{\prime} \max \pm S D\right)$ & $3.01 \pm 0.29$ & $2.72 \pm 0.27$ & 0.72 \\
\hline \multicolumn{4}{|l|}{ Microbiota similarity } \\
\hline Intra-group ${ }^{c}($ mean $\pm \mathrm{SD})$ & $30.47 \pm 9.42$ & $25.86 \pm 11.93$ & 0.001 \\
\hline Inter-group $^{\mathrm{d}}($ mean $\pm \mathrm{SD})$ & $24.13 \pm 10.01$ & - & $<0.001$ \\
\hline
\end{tabular}

Table IV. Analysis of bacterial count by quantitative PCR.

\begin{tabular}{lccc}
\hline Bacteria & $\begin{array}{c}\text { HTN } \\
(\text { mean } \pm \text { SD })\end{array}$ & $\begin{array}{c}\text { CG } \\
(\text { mean } \pm \text { SD) }\end{array}$ & P-value \\
\hline Bacteroides spp. & $6.97 \pm 1.45$ & $6.96 \pm 1.06$ & 0.89 \\
Prevotella spp. $^{\text {a }}$ & $5.36 \pm 2.03$ & $5.08 \pm 1.58$ & 0.04 \\
Clostridium spp. $^{\mathrm{a}}$ & $6.78 \pm 0.79$ & $6.59 \pm 0.38$ & 0.02 \\
Escherichia coli & $5.38 \pm 1.11$ & $4.91 \pm 1.01$ & 0.86 \\
\hline
\end{tabular}

Data are reported as the average estimate of logarithms of fecal PCR target genetic amplicon copy numbers present in $1 \mathrm{~g}$ of feces. ${ }^{\text {a Results that }}$ are significantly different (Student's t-test; $\mathrm{P}<0.05$ ). HTN, hypertension; $\mathrm{CG}$, control group.

different compared with the control group. Triplicate samples were used to calculate the mean within each experiment.

High-throughput sequencing analysis of gene sequences. The comparative statistics of the PCR sequences were estimated using 1,758,974 amplicons at the V3-V4 site of the 16S rRNA gene from 20 patients with hypertension and 10 healthy controls. Among these, the sum of the high-throughput sequencing reads $[1,352,991(860,339$ hypertension and 492,652 healthy control, with an average of 46,654.86 per sample)] passed quality control and were processed for further analysis. The total unique tag counts detected in the hypertension and control groups were 197,167 and 99,191, respectively (with an average of 10,219.24 for all samples). The total number of OTUs assigned was 5,566 (3,653 hypertension and 1,913 healthy control, with an average of 192 per sample). The sum of the unique tags from the two groups was 296,358, which revealed the total phylotypes in the present study, and after deletion of linkage primers, the length of the average sequence was $421 \mathrm{bp}$.

Gut microbial diversity and composition. The richness and diversity of the microbial community were calculated at the $97 \%$ similarity level. Rarefaction curves indicated that the
Table V. Gut bacterial richness and diversity index at $97 \%$ similarity, analyzed by high-throughput sequencing.

\begin{tabular}{lccc}
\hline & \multicolumn{2}{c}{ Group } & \\
\cline { 2 - 3 } Index & HTN & CG & P-value \\
\hline Observed species & 182.6 & 169.7 & 0.3 \\
OTUs & 209.3 & 191.3 & 0.12 \\
Shannon & 4.36 & 4.25 & 0.63 \\
Simpson & 0.89 & 0.88 & 0.88 \\
Chao1 $^{\mathrm{a}}$ & 225.7 & 192.73 & 0.03 \\
ACE $^{\mathrm{a}}$ & 226.1 & 194.98 & 0.02 \\
Good's coverage & 0.9988 & 0.9988 & 1.000 \\
\hline
\end{tabular}

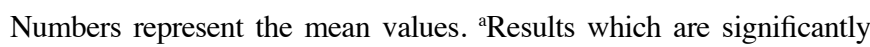
different (Student's t-test; $\mathrm{P}<0.05$ ). HTN, hypertension; CG, control group; OTUs, operational taxonomic units; ACE, alternating conditional expectations.

microbial communities were well represented for most of the samples, and there were no obvious differences in the rarefaction curves between the two groups (Fig. S1). The number (mean) of bacterial OTUs and the observed species were higher in the hypertension group compared with the healthy control group (Table V). Good's coverage was $\sim 99 \%$ for all sequences, indicating that the $16 \mathrm{~S}$ rRNA sequences identified in the two groups were the major bacterial sequences present in the study samples. The $\alpha$-diversity, determined using the nonparametric ACE and Chaol algorithms, was significantly higher in the hypertension group than in the healthy individuals $(\mathrm{P}=0.02$ and $\mathrm{P}=0.03$, respectively). The study samples were divided into two clusters, based on UniFrac metrics (Fig. 2).

Gut microbiota distribution at the phylum level. The bacterial composition was assessed on a taxonomic basis at the phylum, family, genus and species levels, and the taxa that accounted 


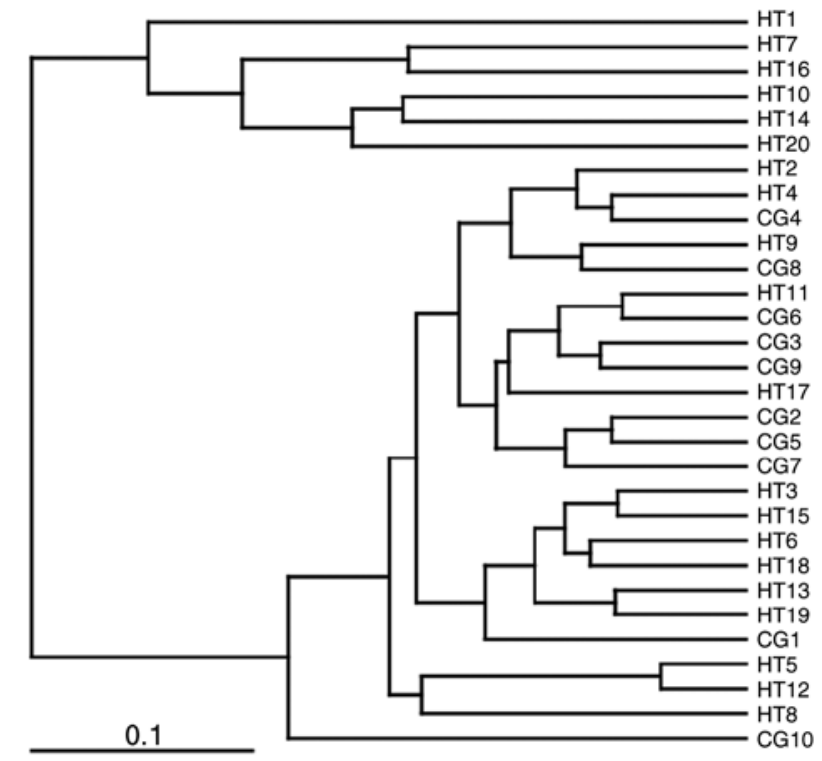

Figure 2. Diversity among fecal samples from patients with hypertension and healthy controls. The unweighted pair group method with the arithmetic mean based on weighted UniFrac distances was used.

for $>1-0.5 \%$ were considered. With respect to the 15 phyla sequenced, the composition of the gut microbiota was different in the two study groups (Fig. 3A). The dominant phyla in all samples were Bacteroidetes (56.58\%), Firmicutes (33.89\%), Proteobacteria (6.74\%) and Actinobacteria (1.99\%), but the only statistically significant quantitative difference between the two groups was in the F/B ratio, which was significantly increased in the hypertension group $(\mathrm{P}=0.02)$ compared with the control group (Fig. 3B). Statistical data of the top 10 phyla are presented in Table SIV. Linear discriminant analysis and effect size measurement was performed, and it was demonstrated that the most differentially abundant bacterial taxa in patients with hypertension were from the phylum Firmicutes (Fig. 4).

Gut microbial distribution at the family level. At the family level, 76 families were sequenced. Among the 10 most prevalent families, Prevotellaceae, Veillonellaceae and Lachnospiraceae were increased in the hypertension group compared with the control group (Fig. 5). In these families, the relative abundance of Bacteroidaceae was lower in patients with hypertension compared with healthy controls. Quantitative differences at the family level are presented in Table SIV.

Gut microbial distribution at the genus level. The high-throughput sequencing identified 175 different genera in the samples. Among the 10 top most prevalent genera, the abundance of Prevotella_9, Megasphaera, Parasutterella and Escherichia-Shigella was significantly increased in the patients with hypertension compared with the healthy control group (Fig. 6). Quantitative differences at the genus level are presented in Table SIV.

Gut microbial distribution at the species level. Faecalibacterium prausnitzii, which accounts for up to $14 \%$ of the total fecal microbiota and has been reported to be the major source of butyrate-producing bacteria (34), was significantly reduced in the hypertension group. Other important butyrate-producing bacterial species in the human colon, such as Roseburia inulinivorans and Anaerostipes hadrus, were reduced in the hypertension group, although not to a significant level. In addition, Bacteroides uniformis was also reduced in the hypertension group compared with the healthy controls, while Phascolarctobacterium faecium was increased in patients with hypertension. No significant differences among Klebsiella spp., Streptococcus spp. and Parabacteroides merdae were observed in our study groups (data not shown). These results suggested that there was considerable dissimilarity between the hypertension and healthy control groups at the species level. The bacterial composition as evaluated by high-throughput sequencing at the species level is presented in Table SV.

Comparative evaluation of analytical techniques used. In the current study, the gut microbiota analysis was performed using three molecular techniques. DGGE and high-throughput sequencing detected the same dominant bacteria, mainly Bacteriodetes and Firmicutes; however, high-throughput sequencing revealed more diversity among bacterial communities and a greater number of phylotypes than PCR-DGGE. The results of qPCR and high-throughput sequencing illustrated the same variations in the composition of the total bacterial community. Overall, the results from the three analytical techniques were in general concordance.

\section{Discussion}

Balance in the gut microbiota is key for maintaining intestinal immunity and homeostasis. A fluctuation in this balance may lead to destructive pathophysiological outcomes (35). Hypertension is the most important, modifiable and potentially reversible risk factor for stroke in all age groups, and is closely associated with SBP and DBP, particularly in patients who experience intracerebral hemorrhage $(36,37)$. The current study focused on molecular characterization of the gut microbiota from patients with hypertension, compared with healthy controls. The compositional characteristics of the gut microbiota in the two groups were determined by PCR-DGGE analysis targeting the $\mathrm{V} 3$ region of the bacterial 16S rRNA gene with imaging and sequencing, qPCR followed by statistical analysis, and high-throughput sequencing of randomly selected samples from the two groups. The $\alpha$-diversity, measured by the nonparametric ACE and Chaol algorithms, was significantly higher in the hypertension group, while the Shannon and Simpson index did not indicate significant differences between the two groups. The gut microbiota distribution was analyzed at the phylum, genus and species levels, and variations between the hypertension and control groups were observed.

Firmicutes, Bacteroidetes and Proteobacteria were the dominant phyla in all the samples, with variable proportions between the two groups. There was a significant difference in the $\mathrm{F} / \mathrm{B}$ ratio between the groups, with an increased ratio in the hypertension group compared with the control group. An increased F/B ratio, due to an increase in Firmicutes or a decline in Bacteroidetes counts, is broadly considered to be an indicator of gut microbiota dysbiosis and is associated with obesity, diabetes mellitus and cardiovascular disease $(15,21,38)$. The $\mathrm{F} / \mathrm{B}$ ratio is well validated in rodent 

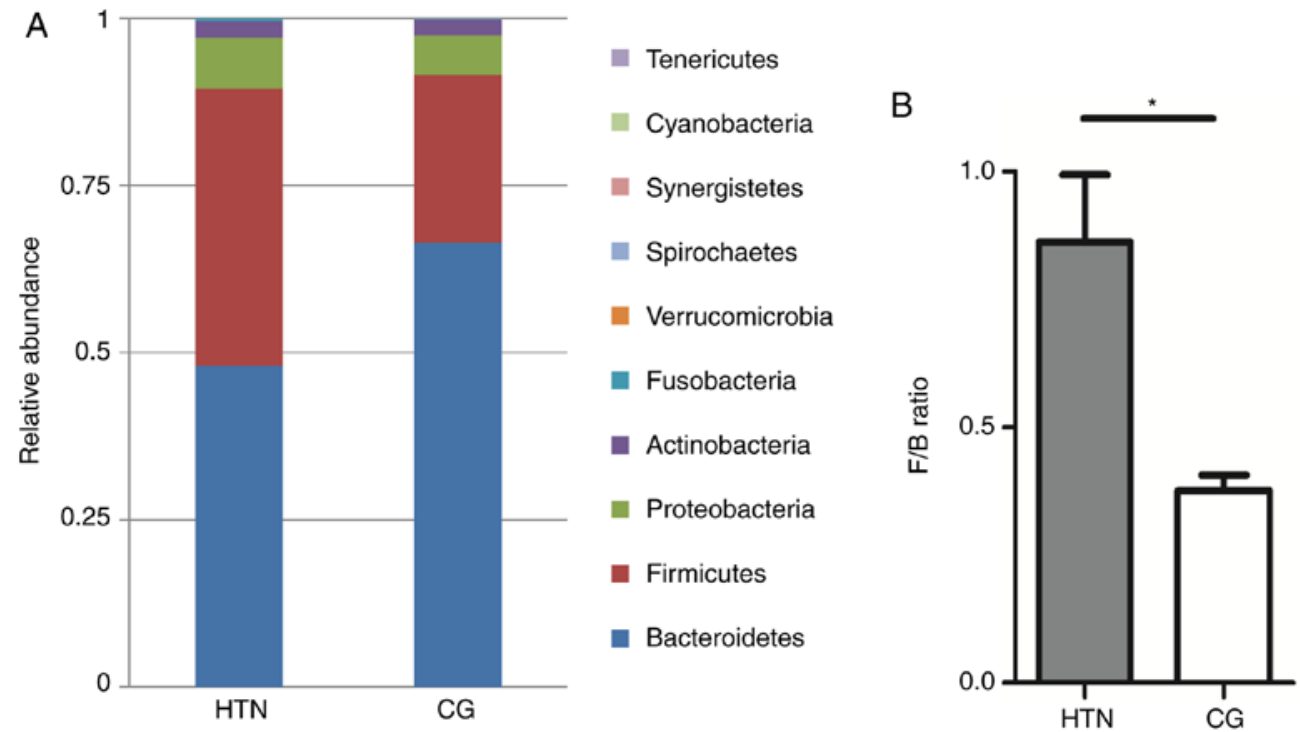

Figure 3. Composition of gut microbiota at the phylum level from high-throughput sequencing. (A) Relative abundances at the phylum level. (B) Statistical analysis of the F/B ratio between the HTN group and CG. "P<0.05. HTN, hypertension; CG, control group; F/B ratio, Firmicutes to Bacteroidetes ratio.

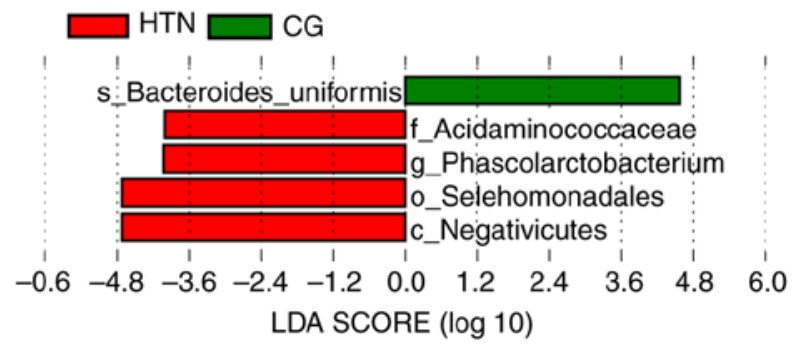

Figure 4. LDA effect size methods were used to identify the most differentially abundant taxa in the HTN group (red) and CG (green). HTN, hypertension; CG, control group; LDA, linear discriminant analysis.

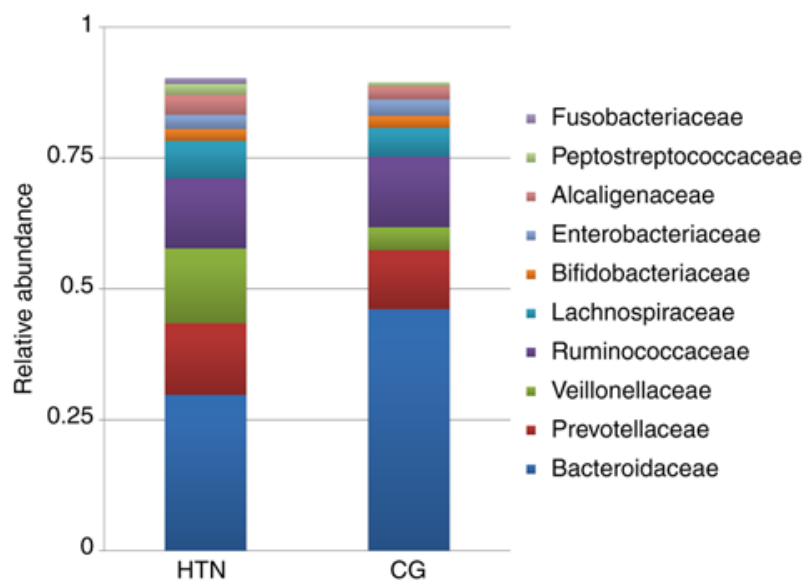

Figure 5. Gut microbial composition analysis at the family level from the high-throughput sequencing results. HTN, hypertension; CG, control group.

and human samples; however, in pig models it requires further confirmation $(39,40)$. These findings clearly indicated the role of the F/B ratio in the pathophysiology of hypertension. Spirochaetes were also increased in the hypertension group; however, this phylum was a very small proportion of the total population $(0.03 \%)$.

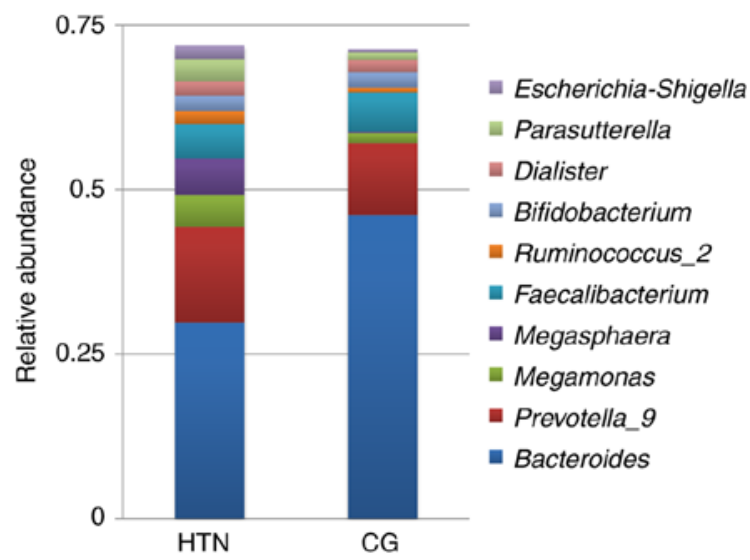

Figure 6. Gut microbiota composition at the genus level analyzed by high-throughput sequencing. HTN, hypertension; CG, control group.

Phascolarctobacterium spp. and Veillonellaceae belong to the same class within the Firmicutes phylum and produce high quantities of short chain fatty acids, acetate and propionate $(41,42)$. The butyrate produced is predominantly used by colonocytes, while acetate and propionate are largely taken up by the liver. Acetate promotes hypercholesterolemia and hypertriglyceridemia, and results in liver steatosis, as it used for cholesterol and fatty acid production (43). Members of the Veillonellaceae family are generally responsible for polymicrobial infections, such as osteoarticular infections or endocarditis, and are rarely associated with monomicrobial infections in humans (44). In the present study, there was an increased abundance of the Veillonellaceae family in patients with hypertension compared with the control group.

At the genus level, there was an increased in the abundance of Prevotella_9, Megasphaera, Parasutterella and Escherichia-Shigella in the hypertension group, as compared with the healthy control group. It has previously been reported that the expansion of Prevotella copri, an intestinal bacterium, is associated with enhanced susceptibility to arthritis $(45,46)$. 
Prevotella copri express a superoxide reductase and phosphoadenosine phosphosulfate reductase that may promote the development of inflammation. Furthermore, Prevotella copri was shown to enhance body weight loss and aggravate epithelial inflammation in a mouse model of colitis (45). Prevotella was overrepresented in individuals with hypertension in the present study, which is consistent with a previous study by Li et al (47), which indicated that these organisms may be increased as a response to hypertension; further investigations may strengthen the association of this bacteria with hypertension. Previous studies have shown that Parasutterella is increased by sugar and alcohol consumption $(48,49)$. An increased abundance of Parasutterella in the gut is associated with dysbiosis, or a lack of microbial diversity $(50,51)$. It has been reported Parasutterella is increased in the submucosa of the ileum in patients with Crohn's disease (50) and in rodents with hypertriglyceridemia-related acute necrotizing pancreatitis (51). However, Parasutterella is a relatively a new discovery, and additional research is required to further establish its role in hypertension and gut microbial dysbiosis. Escherichia-Shigella is closely genetically related to $E$. coli and produces Shiga-toxin that can cause various afflictions, including hemorrhagic colitis, septicemia, severe gastrointestinal tract inflammations in the ileocolonic area, thrombocytopenia, problems in urinary duct channels and hemolytic uremic syndrome (52).

At the species level, the data of the current study demonstrated that Faecalibacterium prausnitzii and Bacteroides uniformis were significantly decreased in the patients with hypertension compared with the healthy control group. Faecalibacterium prausnitzii, the sole known species of the Faecalibacterium genus, is one of the most common gut bacteria, which represents $>10 \%$ of the intestinal microbiota in healthy individuals. It is reported to boost the immune system and serve as an anti-inflammatory agent (53). Low or depleted levels of $F$. prausnitzii are associated with inflammation and observed in a number of diseases, including inflammatory bowel diseases such as Crohn's (54). Increased gut microbial diversity with enrichment of bacterial colonies that produce metabolites such as short-chain fatty acids is favorable and helps to maintain normal physiological homeostasis (5). Faecalibacteria produce butyrate and other short-chain fatty acids through the fermentation of dietary fiber. Butyrate is considered to be one of the most useful short-chain fatty acids that benefits human health via several mechanisms; it reduces the inflammation in adipose tissue and the intestine (55), protects against obesity (induced by food) (56) and cardiovascular disease (57), and improves insulin sensitivity in patients with type 2 diabetes mellitus $(58,59)$. Yan et al (60) reported that Klebsiella spp., Streptococcus spp. and Parabacteroides merdae were frequently distributed in hypertensive gut microbiota, but in our study groups no significant differences among these bacteria were observed. The current study revealed the relative predominance of various microbial populations in the gut at the phylum, genus and species levels in fecal samples from patients with grade 3 hypertension and the healthy controls. The findings illustrate a clear disparity between the study groups, and population comparisons revealed a clear difference in the intestinal microbial populations between the hypertension and healthy control groups.
Limitations of the present study include the difficulty of obtaining the human intestinal contents for microbial community analysis. Ideally, mucosal biopsy samples should be used instead of stool samples, as mucosal microbiota may differ from stool microbiota. However, mucosal sampling presents practical challenges, including disruption of the biofilm when the biopsy is performed and the effect of bowel preparation prior to colonoscopy on the microbiota $(61,62)$. Stool samples are therefore used as a proxy for the study of the gut microbiota as these samples are easier to collect than biopsy samples, especially in healthy volunteers. However, animal experiments need to be performed and the gut microbiota of patients with hypertension may be transplanted into germ-free animals to verify the association of gut dysbiosis with hypertension. Diet is considered another important factor influencing the gut microbial community. In the present study, it was not possible to evaluate the relationship between the gut microbiota and dietary habits of the participants because of the incomplete nutritional information. However, the conclusions are unlikely to be affected by this potential confounding factor, due to likely similar dietary habits and lifestyles of the enrolled subjects from Xi'an, China.

The findings from the DGGE and high-throughput sequencing were consistent and stable. PCR-DGGE is a useful molecular fingerprinting method to qualitatively analyze bacterial communities, especially in the identification of dominant bacteria within a sample. DGGE is fast and multiple samples can be analyzed simultaneously (63). However, it is a semi-quantitative technique and does not give direct phylogenetic identification; thus, it is not optimized for numerical assessment of gut microbiota components (64). When qPCR is used in combination with DGGE, it can provide more detailed information on the diversity and abundance of the gut microbiota (65-67). Metagenomics is the most recent development in the study of the gut microbiota. It is a more sensitive, advanced and authenticated technique, and may be more suitable for examining the microbial ecology. Despite being comparatively expensive, metagenomic techniques have the advantages that they are high throughput, phylogenetically characterize the microbiota components, and quantify the relative proportions of organisms present. On the other hand, PCR-DGGE analysis may be useful as a preliminary test to analyze larger shifts in the bacterial community, as it is economical and less time consuming than high-throughput sequencing.

In conclusion, the findings of current study revealed that the gut microbiota composition was different in the patients with hypertension compared with the healthy control group. More specifically, there was a significant difference in the similarity of bacterial populations, with a significantly increased F/B ratio in the patients with hypertension. It was concluded that hypertension-associated gut dysbiosis is characterized by an imbalance in the normal gut flora. Understanding the fluctuations in microbial composition, and the F/B ratio in particular, may help to identify potential biomarkers for hypertension and other related diseases.

\section{Acknowledgements}

The authors would like to thank Dr Lei Jine (Department of Pathology, First Affiliated Hospital of Xi'an Jiaotong University) for providing the samples from the grade 3 hypertensive patients. The authors are indebted to the Center for 
Disease Control and Prevention of Shaanxi Province for the equipment and technical support.

\section{Funding}

This project was funded by the National Natural Science Foundation of China (grant no. NSFC81730056).

\section{Availability of data and materials}

The datasets used and/or analyzed during the current study are available from the corresponding author on reasonable request.

\section{Authors' contributions}

NM designed and executed the experiments, analyzed the data and wrote the manuscript. SH helped in the analyses and interpretation of data. SZ, LY, HL, SU, YW helped perform the experiments. JX conceived and designed the study, critically reviewed and drafted the manuscript and take responsibility for the integrity of the work as a whole. All authors read and approved the final manuscript.

\section{Ethics approval and consent to participate}

Informed written consent was obtained from all participants in the study. The present study was performed with the approval of the Ethical Committee of Xi'an Jiaotong University School of Medical Sciences, under the guidelines of the World Medical Association and the Declaration of Helsinki.

\section{Patient consent for publication}

Informed written consent for publication was obtained from all the participants.

\section{Competing interests}

The authors declare that they have no competing interests.

\section{References}

1. Lim SS, Vos T,Flaxman AD, Danaei G, Shibuya K, Adair-RohaniH, Amann M, Anderson HR, Andrews KG, Aryee M, et al: A comparative risk assessment of burden of disease and injury attributable to 67 risk factors and risk factor clusters in 21 regions, 1990-2010: A systematic analysis for the global burden of disease study 2010. Lancet 380: 2224-2260, 2012.

2. Lackland DT and Weber MA: Global burden of cardiovascular disease and stroke: Hypertension at the core. Can J Cardiol 31: 569-571, 2015.

3. Mills KT, Bundy JD, Kelly TN, Reed JE, Kearney PM, Reynolds K, Chen J and He J: Global disparities of hypertension prevalence and control: A systematic analysis of population-based studies from 90 countries. Circulation 134: 441-450, 2016.

4. Durgan DJ, Ganesh BP, Cope JL, Ajami NJ, Phillips SC, Petrosino JF, Hollister EB and Bryan RM Jr: Role of the gut microbiome in obstructive sleep apnea-induced hypertension. Hypertension 67: 469-474, 2016.

5. Yang T,Santisteban MM,Rodriguez V,LiE,Ahmari N,Carvajal JM, Zadeh M, Gong M, Qi Y,Zubcevic J, et al: Gut dysbiosis is linked to hypertension. Hypertension 65: 1331-1340, 2015.

6. Mell B, Jala VR, Mathew AV, Byun J, Waghulde H, Zhang Y, Haribabu B, Vijay-Kumar M, Pennathur S and Joe B: Evidence for a link between gut microbiota and hypertension in the Dahl rat. Physiol Genomics 47: 187-197, 2015.
7. Nutting CW, Islam S and Daugirdas JT: Vasorelaxant effects of short chain fatty acid salts in rat caudal artery. Am J Physiol 261: H561-H567, 1991.

8. Pluznick JL, Zou DJ, Zhang X, Yan Q, Rodriguez-Gil DJ, Eisner C, Wells E, Greer CA, Wang T, Firestein S, et al: Functional expression of the olfactory signaling system in the kidney. Proc Natl Acad Sci USA 106: 2059-2064, 2009.

9. Galla S, Chakraborty S, Mell B, Vijay-Kumar M and Joe B: Microbiotal-host interactions and hypertension. Physiology (Bethesda) 32: 224-233, 2017.

10. Menni C, Graham D, Kastenmuller G, Alharbi NH, Alsanosi SM, McBride M, Mangino M, Titcombe P, Shin SY, Psatha M, et al: Metabolomic identification of a novel pathway of blood pressure regulation involving hexadecanedioate. Hypertension 66: 422-429, 2015.

11. Thursby $\mathrm{E}$ and Juge $\mathrm{N}$ : Introduction to the human gut microbiota. Biochem J 474: 1823-1836, 2017.

12. Sekirov I, Russell SL, Antunes LC and Finlay BB: Gut microbiota in health and disease. Physiol Rev 90: 859-904, 2010.

13. Sanz Y and Moya-Perez A: Microbiota, inflammation and obesity. Adv Exp Med Biol 817: 291-317, 2014.

14. Mariat D, Firmesse O, Levenez F, Guimarăes V, Sokol H, Doré J, Corthier G and Furet JP: The firmicutes/bacteroidetes ratio of the human microbiota changes with age. BMC Microbiol 9: 123, 2009.

15. Khalesi S, Sun J, Buys N and Jayasinghe R: Effect of probiotics on blood pressure: A systematic review and meta-analysis of randomized, controlled trials. Hypertension 64: 897-903, 2014.

16. Mann GV: Studies of a surfactant and cholesteremia in the Maasai. Am J Clinl Nutr 27: 464-469, 1974.

17. Kiessling G, Schneider J and Jahreis G: Long-term consumption of fermented dairy products over 6 months increases HDL cholesterol. Eur J Clin Nutr 56: 843-849, 2002.

18. Collado MC, Rautava S, Isolauri E and Salminen S: Gut microbiota: A source of novel tools to reduce the risk of human disease? Pediatr Res 77: 182-188, 2015.

19. Frank DN, St Amand AL, Feldman RA, Boedeker EC, Harpaz N and Pace NR: Molecular-phylogenetic characterization of microbial community imbalances in human inflammatory bowel diseases. Proc Natl Acad Sci USA 104: 13780-13785, 2007.

20. Qin J, Li R, Raes J, Arumugam M, Burgdorf KS, Manichanh C, Nielsen T, Pons N, Levenez F, Yamada T, et al: A human gut microbial gene catalogue established by metagenomic sequencing. Nature 464: 59-65, 2010.

21. Ley RE, Turnbaugh PJ, Klein S and Gordon JI: Microbial ecology: Human gut microbes associated with obesity. Nature 444: 1022-1023, 2006

22. Kau AL, Ahern PP, Griffin NW, Goodman AL and Gordon JI: Human nutrition, the gut microbiome and the immune system. Nature 474: 327-336, 2011.

23. Muyzer G, de Waal EC and Uitterlinden AG: Profiling of complex microbial populations by denaturing gradient gel electrophoresis analysis of polymerase chain reaction-amplified genes coding for 16S rRNA. Appl Environ Microbiol 59: 695-700, 1993.

24. Fromin N, Hamelin J, Tarnawski S, Roesti D, Jourdain-Miserez K, Forestier N, Teyssier-Cuvelle S, Gillet F, Aragno M and Rossi P: Statistical analysis of denaturing gel electrophoresis (DGE) fingerprinting patterns. Environ Microbiol 4: 634-643, 2002.

25. Lu Y, Xie L and Chen J: A novel procedure for absolute real-time quantification of gene expression patterns. Plant Methods 8: 9 , 2012.

26. Sun H, Tang JW, Fang CL, Yao XH, Wu YF, Wang X and Feng J: Molecular analysis of intestinal bacterial microbiota of broiler chickens fed diets containing fermented cottonseed meal. Poult Sci 92: 392-401, 2013.

27. Peiffer JA, Spor A, Koren O, Jin Z, Tringe SG, Dangl JL, Buckler ES and Ley RE: Diversity and heritability of the maize rhizosphere microbiome under field conditions. Proc Natl Acad Sci USA 110: 6548-6553, 2013.

28. Caporaso JG, Kuczynski J, Stombaugh J, Bittinger K, Bushman FD, Costello EK, Fierer N, Peña AG, Goodrich JK, Gordon JI, et al: QIIME allows analysis of high-throughput community sequencing data. Nat Methods 7: 335-336, 2010.

29. Magoc T and Salzberg SL: FLASH: Fast length adjustment of short reads to improve genome assemblies. Bioinformatics 27: 2957-2963, 2011.

30. DeSantis TZ, Hugenholtz P, Larsen N, Rojas M, Brodie EL, Keller K, Huber T, Dalevi D, Hu P and Andersen GL: Greengenes, a chimera-checked 16S rRNA gene database and workbench compatible with ARB. Appl Environ Microbiol 72: 5069-5072, 2006.

31. Edgar RC: Search and clustering orders of magnitude faster than BLAST. Bioinformatics 26: 2460-2461, 2010. 
32. Wang Q, Garrity GM, Tiedje JM and Cole JR: Naive bayesian classifier for rapid assignment of rRNA sequences into the new bacterial taxonomy. App Environ Microbiol 73: 5261-5267, 2007.

33. Team RC: R: A language and environment for statistical computing. R foundation for statistical computing, Vienna, Austria, 2012.

34. De Vuyst L, Moens F, Selak M, Riviere A and Leroy F: Summer meeting 2013: Growth and physiology of bifidobacteria. J Appl Microbiol 116: 477-491, 2014.

35. Kearney PM, Whelton M, Reynolds K, Whelton PK and He J: Worldwide prevalence of hypertension: A systematic review. J Hypertens 22: 11-19, 2004.

36. Arima H, Chalmers J, Woodward M, Anderson C, Rodgers A, Davis S, Macmahon S and Neal B; PROGRESS Collaborative Group: Lower target blood pressures are safe and effective for the prevention of recurrent stroke: The PROGRESS trial. J Hyperten 24: 1201-1208, 2006.

37. Goldstein LB, Adams R, Alberts MJ, Appel LJ, Brass LM, Bushnell CD, Culebras A, Degraba TJ, Gorelick PB, Guyton JR, et al: Primary prevention of ischemic stroke: A guideline from the american heart association/American stroke association stroke council: Cosponsored by the atherosclerotic peripheral vascular disease interdisciplinary working group; cardiovascular nursing council; clinical cardiology council; nutrition, physical activity, and metabolism council; and the quality of care and outcomes research interdisciplinary working group: The American academy of neurology affirms the value of this guideline. Stroke 37: 1583-1633, 2006.

38. Tilg $\mathrm{H}$ and Kaser A: Gut microbiome, obesity, and metabolic dysfunction. J Clin Invest 121: 2126-2132, 2011.

39. Duca FA, Sakar Y, Lepage P, Devime F, Langelier B, Doré J and Covasa M: Replication of obesity and associated signaling pathways through transfer of microbiota from obese-prone rats. Diabetes 63: 1624-1636, 2014.

40. Pedersen R, Andersen AD, Molbak L, Stagsted J and Boye M: Changes in the gut microbiota of cloned and non-cloned control pigs during development of obesity: Gut microbiota during development of obesity in cloned pigs. BMC Microbiol 13: 30, 2013.

41. Duncan SH, Holtrop G, Lobley GE, Calder AG, Stewart CS and Flint HJ: Contribution of acetate to butyrate formation by human faecal bacteria. Br J Nutr 91: 915-923, 2004

42. Watanabe Y, Nagai F and Morotomi M: Characterization of Phascolarctobacterium succinatutens sp. Nov.an asaccharolytic, succinate-utilizing bacterium isolated from human feces. Appl Environ Microbiol 78: 511-518, 2012.

43. Wong JM, de Souza R, Kendall CW, Emam A and Jenkins DJ: Colonic health: Fermentation and short chain fatty acids. J Clin Gastroenterol 40: 235-243, 2006.

44. Marchandin H and Jumas-Bilak E: The family Veillonellaceae: The prokaryotes: Firmicutes and tenericutes. Rosenberg E DeLong EF, Lory S, Stackebrandt E and Thompson F (eds). Springer Berlin Heidelberg, Berlin, Heidelberg, pp433-453, 2014

45. Hofer U: Microbiome: Anelloviridae go viral. Nat Rev Microbiol 12: 4-5, 2014

46. Scher JU, Sczesnak A, Longman RS, Segata N, Ubeda C Bielski C, Rostron T, Cerundolo V, Pamer EG, Abramson SB, et al: Expansion of intestinal Prevotella copri correlates with enhanced susceptibility to arthritis. ELife 2: e01202, 2013.

47. Li J, Zhao F, Wang Y, Chen J, Tao J, Tian G, Wu S, Liu W, Cui Q, Geng B, et al: Gut microbiota dysbiosis contributes to the development of hypertension. Microbiome 5: 14, 2017.

48. Zhang X, Wang H, Yin P, Fan H, Sun L and Liu Y: Flaxseed oil ameliorates alcoholic liver disease via anti-inflammation and modulating gut microbiota in mice. Lipids Health Dis 16: 44, 2017

49. Noble EE, Hsu TM, Jones RB, Fodor AA, Goran MI and Kanoski SE: Early-life sugar consumption affects the rat microbiome independently of obesity. J Nutr 147: 20-28, 2017

50. Chiodini RJ, Dowd SE, Chamberlin WM, Galandiuk S, Davis B and Glassing A: Microbial population differentials between mucosal and submucosal intestinal tissues in advanced crohn's disease of the ileum. PLoS One 10: e0134382, 2015.

51. Huang C, Chen J, Wang J, Zhou H, Lu Y, Louz L, Zheng J, Tian L, Wang X, Cao Z and Zeng Y: Dysbiosis of intestinal microbiota and decreased antimicrobial peptide level in paneth cells during hypertriglyceridemia-related acute necrotizing pancreatitis in rats. Front Microbiol 8: 776, 2017.

52. Amani J, Ahmadpour A, Imani Fooladi AA and Nazarian S: Detection of E. coli $\mathrm{O} 157: \mathrm{H} 7$ and Shigella dysenteriae toxins in clinical samples by PCR-ELISA. Braz J Infect Dis 19: 278-284, 2015 .
53. Miquel S, Martin R, Rossi O, Bermúdez-Humarán LG, Chatel JM, Sokol H, Thomas M, Wells JM and Langella P: Faecalibacterium prausnitzii and human intestinal health. Curr Opin Microbiol 16: 255-261, 2013

54. Cao Y, Shen J and Ran ZH: Association between Faecalibacterium prausnitzii reduction and inflammatory bowel disease: A meta-analysis and systematic review of the literature. Gastroenterol Res Pract 2014: 872725, 2014

55. Vinolo MA, Rodrigues HG, Nachbar RT and Curi R: Regulation of inflammation by short chain fatty acids. Nutrients 3: 858-876, 2011.

56. Lin HV, Frassetto A, Kowalik EJ Jr, Nawrocki AR, Lu MM, Kosinski JR, Hubert JA, Szeto D, Yao X, Forrest G and Marsh DJ: Butyrate and propionate protect against diet-induced obesity and regulate gut hormones via free fatty acid receptor 3-independent mechanisms. PLoS One 7: e35240, 2012.

57. Berni Canani R, Di Costanzo M and Leone L: The epigenetic effects of butyrate: Potential therapeutic implications for clinical practice. Clin Epigenetics 4: 4, 2012.

58. Henagan TM, Stefanska B, Fang Z, Navard AM, Ye J, Lenard NR and Devarshi PP: Sodium butyrate epigenetically modulates high-fat diet-induced skeletal muscle mitochondrial adaptation, obesity and insulin resistance through nucleosome positioning. Br J Pharmacol 172: 2782-2798, 2015.

59. Gao Z, Yin J, Zhang J, Ward RE, Martin RJ, Lefevre M, Cefalu WT and Ye J: Butyrate improves insulin sensitivity and increases energy expenditure in mice. Diabetes 58: 1509-1517, 2009.

60. Yan Q, Gu Y, Li X, Yang W, Jia L, Chen C, Han X, Huang Y, Zhao L, Li P, et al: Alterations of the gut microbiome in hypertension. Front Cell Infect Microbiol 7: 381, 2017.

61. Zoetendal EG, von Wright A, Vilpponen-Salmela T, Ben-Amor K, Akkermans AD and de Vos WM: Mucosa-associated bacteria in the human gastrointestinal tract are uniformly distributed along the colon and differ from the community recovered from feces. Appl Environ Microbiol 68: 3401-3407, 2002

62. Eckburg PB, Bik EM, Bernstein CN, Purdom E, Dethlefsen L, Sargent M, Gill SR, Nelson KE and Relman DA: Diversity of the human intestinal microbial flora. Science 308: 1635-1638, 2005.

63. Muyzer G: DGGE/TGGE a method for identifying genes from natural ecosystems. Curr Opin Microbiol 2: 317-322, 1999.

64. Rinttila T, Kassinen A, Malinen E, Krogius L and Palva A: Development of an extensive set of $16 \mathrm{~S}$ rDNA-targeted primers for quantification of pathogenic and indigenous bacteria in faecal samples by real-time PCR. J Appl Microbiol 97: 1166-1177, 2004.

65. Ponnusamy K, Choi JN, Kim J, Lee SY and Lee CH: Microbial community and metabolomic comparison of irritable bowel syndrome faeces. J Med Microbiol 60: 817-827, 2011.

66. Jalanka-Tuovinen J, Salonen A, Nikkila J, Immonen O, Kekkonen R, Lahti L, Palva A and de Vos WM: Intestinal microbiota in healthy adults: Temporal analysis reveals individual and common core and relation to intestinal symptoms. PLoS One 6: e23035, 2011.

67. Zwielehner J, Liszt K, Handschur M, Lassl C, Lapin A and Haslberger AG: Combined PCR-DGGE fingerprinting and quantitative-PCR indicates shifts in fecal population sizes and diversity of Bacteroides, bifidobacteria and Clostridium cluster IV in institutionalized elderly. Exp Gerontol 44: 440-446, 2009.

68. Huijsdens XW, Linskens RK, Mak M, Meuwissen SG Vandenbroucke-Grauls CM and Savelkoul PH: Quantification of bacteria adherent to gastrointestinal mucosa by real-time PCR J Clin Microbiol 40: 4423-4427, 2002

69. Matsuki T, Watanabe K, Fujimoto J, Miyamoto Y, Takada T, Matsumoto K, Oyaizu H and Tanaka R: Development of 16S rRNA-gene-targeted group-specific primers for the detection and identification of predominant bacteria in human feces. Appl Environ Microbiol 68: 5445-5451, 2002.

70. Bartosch S, Fite A, Macfarlane GT and McMurdo ME: Characterization of bacterial communities in feces from healthy elderly volunteers and hospitalized elderly patients by using real-time PCR and effects of antibiotic treatment on the fecal microbiota. Appl Environ Microbiol 70: 3575-3581, 2004

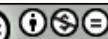

This work is licensed under a Creative Commons Attribution-NonCommercial-NoDerivatives 4.0 International (CC BY-NC-ND 4.0) License. 